\title{
Local and Global Lyapunov Exponents in a Discrete Mass Waterwheel
}

\author{
David Becerra Alonso and Valery Tereshko
}

School of Computing

University of the West of Scotland

Paisley PA1 2BE

United Kingdom

(e-mail: David.Becerra.Alonso, Valery.Tereshko@uws.ac.uk)

\begin{abstract}
A generic Jacobian is calculated to obtain the Lyapunov exponents Malkus' system. However complete, the Lyapunov exponents obtained from the Jacobian do not appropriately show the distinction between chaos and order. A further explanation for this is required. We show how the waterwheel equations, chaotic as a whole, can be decomposed into a series of convergent equations. Chaos will then come in from the transition between any two of these convergent equations. We finally use a common numerical method, not based on the Jacobian, to obtain Lyapunov exponents that properly make the distinction between chaos and order.
\end{abstract}

\section{Introduction}

The equations of motion for Malkus' waterwheel, obtained via analytical mechanics, were presented in [1]. The proper derivation into the Lorenz system [4] has been supported in a series of publications since the waterwheel was first proposed by Malkus in 1972 [5]. Among these it is worth citing [3], in which the Lorenz equations are obtained using a Fourier solution for the distribution of mass over the rim of the waterwheel. The equations for the position $(\theta)$ and angular velocity $(\omega)$ of the waterwheel are given by:

$$
\begin{gathered}
\dot{\theta}=\omega \\
\dot{\omega}=\frac{g R \sum_{i=0}^{N-1} m_{i} \sin \left(\theta+i \frac{2 \pi}{N}\right)-\nu \omega}{I_{0}+R^{2} \sum_{i=0}^{N-1} m_{i}}
\end{gathered}
$$

where $R$ is the radius of the wheel, $g$ is gravity, $N$ is the number of buckets, $\nu$ is the friction coefficient, $I_{0}$ is the moment of inertia when no water has yet been poured on the waterwheel, and $m_{i}$ are the masses of water on each bucket at any given moment. All of them are parameters, except $m_{i}$ which also requires its own differential equation:

$$
\dot{m}_{i}=q(\theta)-k m_{i}
$$

where

$$
q(\theta)= \begin{cases}\tilde{q} & \text { if }\left|\theta+i \frac{2 \pi}{N}\right| \leq \arcsin \frac{l}{2 R} \\ 0 & \text { otherwise }\end{cases}
$$


Becerra and Tereshko

\section{ORDER-CHAOS-ORDER TRANSITIONS}

Here we assumed that the water jet is narrower than the bucket size $l$, so that the latter completely defines the angle at which a bucket is exposed to the water. In (3), $q$ represents the amount of incoming water per second and $k$ is the leak rate of each one of the buckets.

\section{Order-chaos-order transitions}

In order to present a straightforward scenario of chaotic and periodic regimes being clearly differentiated, we plot a bifurcation diagram where we fix all parameters except $\tilde{q}$. As cut condition, we will extract the values of $\omega$ every time a specific bucket goes through to top of the wheel $(\theta=0)$.

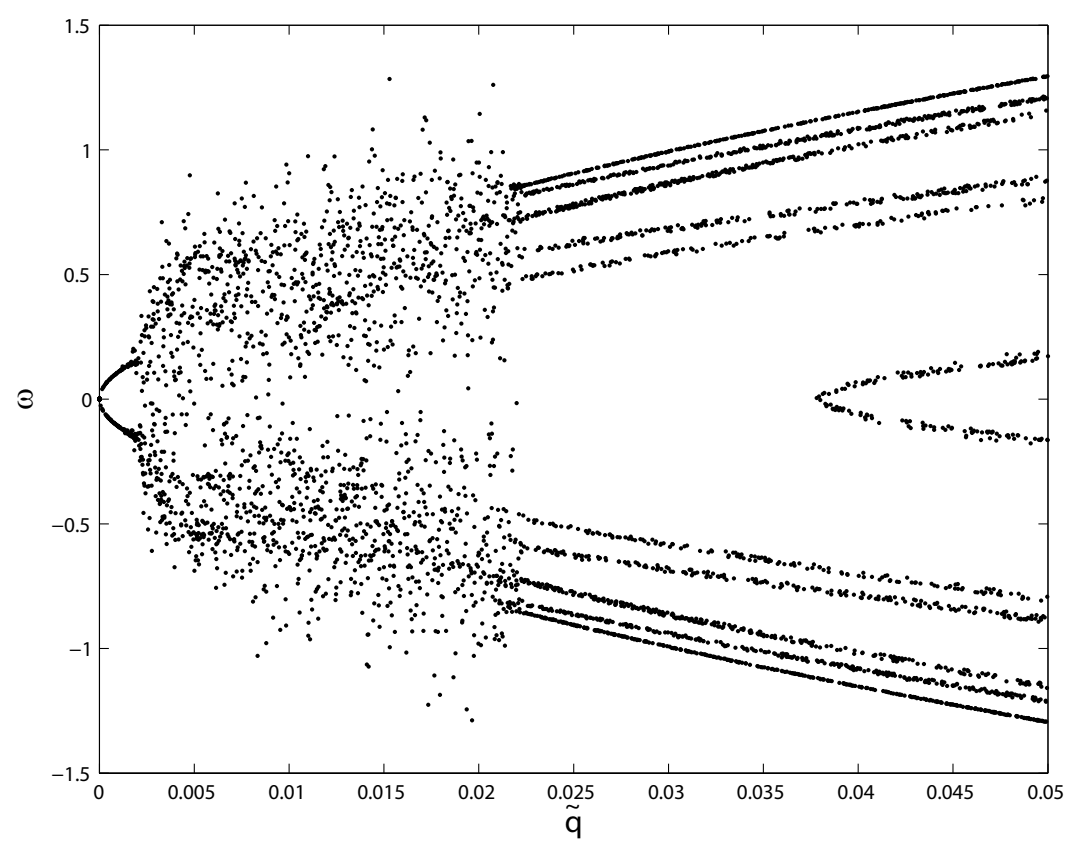

Fig. 1. Bifurcation diagram with $\tilde{q}$ as bifurcation parameter, where $k=0.013$, $I_{0}=0.04, \nu=1.5, R=2, g=9.8$ and $N=8$.

The case for $N=8$ will be representative enough for the purpose of this paper. We have found that the order-chaos-order transition values of $\tilde{q}$ settle to values very close to the ones found for $N=8$ as we keep on increasing the number of buckets.

In this particular case we can identify four very well differentiated regimes: 
- $\tilde{q}=0 \longrightarrow$ Trivial stationary state.

- $\tilde{q} \in(0,0.002) \longrightarrow$ Periodic rotation of the waterwheel, always in the same direction. $+\omega$ values are for clockwise, and $-\omega$ for counterclockwise.

- $\tilde{q} \in(0.002,0.022) \longrightarrow$ Chaotic regime.

- $\tilde{q}>0.022 \longrightarrow$ Periodic regime where the waterwheel repeatedly goes once clockwise and once counterclockwise. Within this regime we can easily identify 6 different frequencies. The waterwheel will settle on one of these frequencies depending on the initial conditions.

As part of our analysis we wanted to look at the Lyapunov exponents for this system, as a means to systematically differentiate chaos from the other regimes. The idea was then to calculate the Jacobian, the eigenvalues, obtain the local Lyapunov exponents for a long enough time lapse, and average those to obtain a global Lyapunov exponent.

\section{Approximated piecewise Jacobian}

All the elements of the differential equations are derivable except $q(\theta)$. The derivative of $q(\theta)$ is always zero except in the moments when a bucket stops being the highest one in favor of another bucket. In those moments the derivative of $q(\theta)$ instantaneously peaks. But of course if we are going to calculate the global Lyapunov exponent as the average of all the local ones, these particular peaks would not affect this average compared to all the $\theta \mathrm{s}$ where no transition between buckets takes place.

This means that for the purpose of calculating the global Lyapunov exponent based on the average of the locals, we can propose a simplified Jacobian where the derivatives of $q$ with respect to $\theta$ remain equal to zero at all times. The Jacobian for equations (1), (2) and (3), and for $\mathrm{N}$ buckets would be an $(N+2) \times(N+2)$ matrix of the form:

$$
\mathbf{J}=\left(\begin{array}{cccccc}
0 & 1 & 0 & 0 & \ldots & 0 \\
d_{2,1} & d_{2,2} & d_{2,3} & d_{2,4} & \ldots & d_{2,(N+2)} \\
d_{3,1} & 0 & -k & 0 & \ldots & 0 \\
d_{4,1} & 0 & 0 & -k & \ldots & 0 \\
\vdots & \vdots & \vdots & \vdots & \ddots & \vdots \\
d_{(N+2), 1} & 0 & 0 & 0 & \ldots & -k
\end{array}\right)
$$

where

$$
\begin{gathered}
d_{2,1}=\frac{g R \sum_{i} m_{i} \cos \left(\theta+i \frac{2 \pi}{N}\right)}{I_{0}+R^{2} \sum_{i} m_{i}} \\
d_{2,2}=\frac{-\nu}{I_{0}+R^{2} \sum_{i} m_{i}}
\end{gathered}
$$




$$
\begin{gathered}
d_{2,(j \geq 3)}=\frac{g R \sin \left(\theta+(j-2) \frac{2 \pi}{N}\right)}{I_{0}+R^{2} \sum_{i} m_{i}}-\frac{R^{2}\left(-\nu \omega+g R \sum_{i} m_{i} \sin \left(\theta+i \frac{2 \pi}{N}\right)\right)}{\left(I_{0}+R^{2} \sum_{i} m_{i}\right)^{2}} \\
d_{(j \geq 3), 1}=0
\end{gathered}
$$

The elements $d_{(j \geq 3), 1}$ would be different from zero in the unsimplified case.

\section{Jacobian-based local Lyapunov exponents}

We expect the values of $\tilde{q}$ within the chaotic regime to be positive. For the numerical test, we choose $\tilde{q}=0.02$. After ensuring that the transient is over, these are the temporal dynamics of $\omega(t)$ and the local Lyapunov exponents.
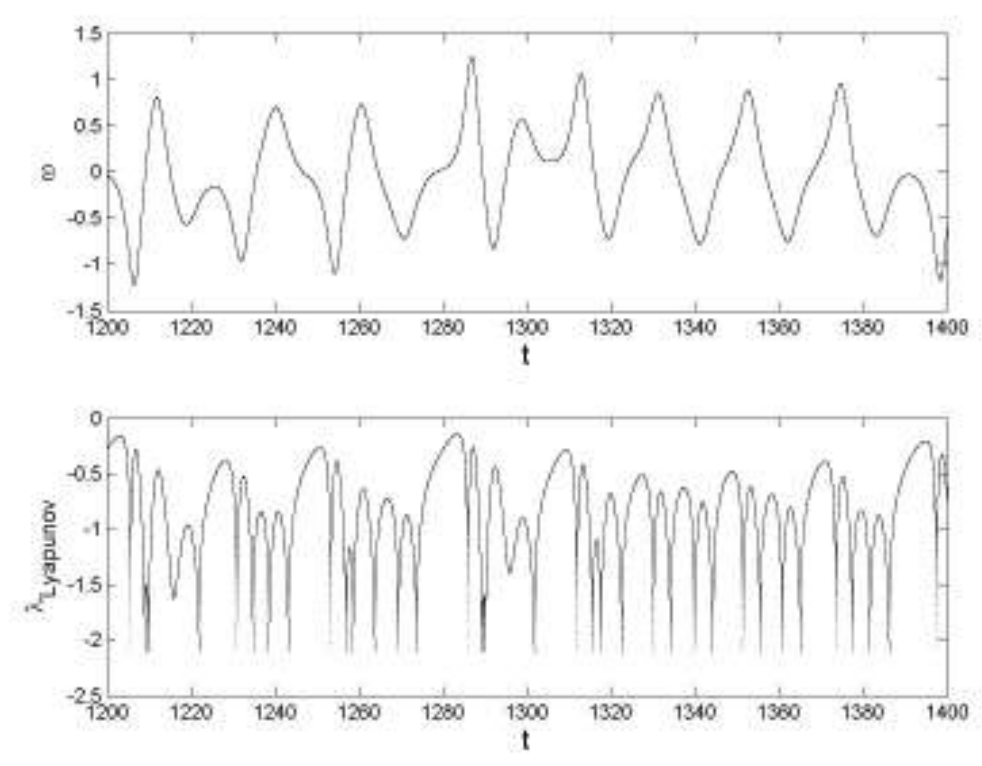

Fig. 2. $\omega$ and Local Lyapunov exponents vs. time for $\tilde{q}=0.02$. All other parameters remain as above.

Two consequences can be inferred from this result. The most direct is that the average of $\lambda_{\text {Lyapunov }}$ is clearly negative. We will go into detail about this on the next section.

The second is that we can find an inverse proportionality between angular acceleration and the local Lyapunov exponents. Every time $\omega$ hits an extreme or goes through an inflexion point, there is a corresponding peak in 
the temporal dynamics of $\lambda_{\text {Lyapunov }}$. As we can see in Figure 2, the more significant peaks are those when $\omega$ is going through a low slope inflexion point or an extreme of $\omega$ happens close to $\omega=0$.

This has an equivalent physical explanation when we think about the behaviour of the waterwheel. Higher local Lyapunov exponents imply a higher sensitive dependence to initial conditions at that particular state of the system. Malkus' waterwheel was expected to have different sensitivity depending on $\omega$. When $\omega$ is close to zero, the waterwheel is slowing down, and the sum of the torques exerted by the water on each one of the buckets will decide if it keeps rotating in the same direction or not. In such a tipping point, very different future states of the system are being decided, and therefore the sensitivity to any perturbation is high. One of the things that makes Malkus' waterwheel a challenging model is that predictions beyond any of these tipping points are very difficult, and these are happening just a few seconds apart from each other.

\section{Reasons for $\lambda_{\text {local }}<0$ in chaos}

The local Lyapunov exponents calculated in the previous section were the result of obtaining the eigenvalues of the Jacobian, calculating the absolute values, choosing the highest one, and applying the natural logarithm. This goes with the standard definition of Lyapunov exponent. However, the Jacobian as a derivative has a very strong limitation in this particular system when we try to use it as a means to measure the long term butterfly effect. Our equations for the waterwheel, as we presented them in Section 1, are piecewise because of $q(\theta)$. Though the complete system presents nonlinearity and chaos (with the right parameters), each one of the individual piecewise subsystems is convergent.

This can also be translated into a physical explanation: as long as the top faucet keeps on filling the same bucket while the others drain, the system will be converging. At the very moment when a new bucket is the one under the faucet, the convergent state of the system is displaced, and the system will start approaching that new convergent state. It's this permanent shift of stationary states that keeps the waterwheel going.

But of course that means that our derivatives are those of a convergent subsystem, part of the complete piecewise system. This makes it natural for the corresponding Lyapunov exponents to be negative. Even if we had not proposed the simplified Jacobian, the result would remain the same, because the difference would only be relevant during transitions between buckets, while the rest of the local Lyapunov exponents stay negative. It is the long term permanent transition between buckets that eventually allows the butterfly effect to take over, and no local and short term analysis will show this.

In Figure 3 we can see the temporal dynamics of the system under two initial conditions separated by a distance $D_{0}=10^{-11}$. Two stages are eas- 

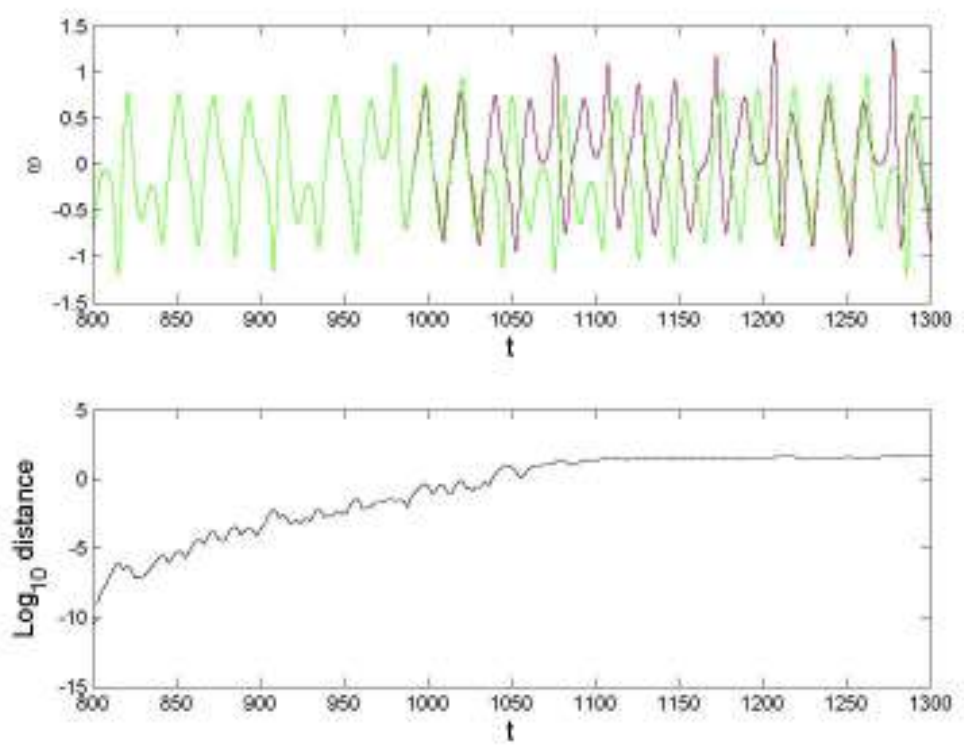

Fig. 3. Butterfly effect for $\tilde{q}=0.02$. All other parameters remain as above.

ily recognized. While the distance $D$ between the two systems is less than the width of a bucket, the decimal logarithm of the distance increases in an approximately linear way. The moment we have $D$ larger than the width of a bucket, divergence is guaranteed because each system is governed by a different piecewise subsystem. This is when the butterfly effect saturates.

In Figure 4 we choose a $\tilde{q}$ that corresponds to a periodic state. The distance between the two initial conditions is a few orders of magnitude below the width of a bucket. So they are both under the same piecewise subsystem at practically all times. Thus, saturation of the distance happens a lot sooner and divergence never takes place.

\section{Global Lyapunov exponents}

Since the Jacobian-based Lyapunov exponents are not going to be good for the distinction between chaotic and periodic states, we will need to use a different method. The numerical method we will use, can be found in detail in $[6,2]$. In this case, the method converges to the global Lyapunov Exponent instead of being obtained as the averages of the locals. For all the reasons presented in Section 5, it takes some time for this convergence to take place.

Figure 5 shows that the numerical method for global Lyapunov exponents 

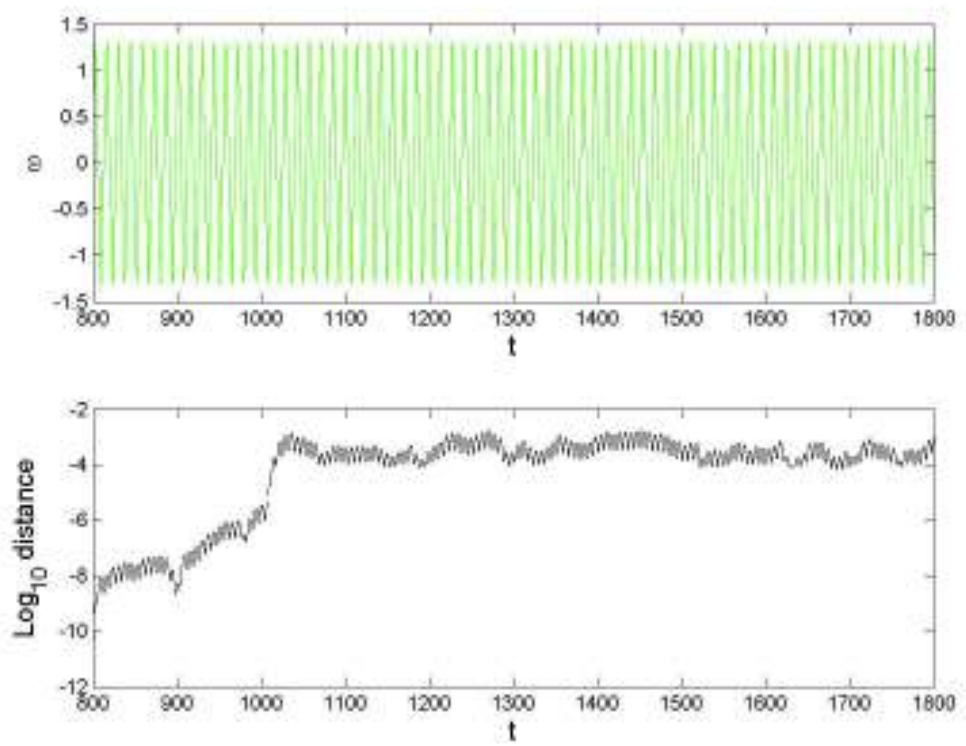

Fig. 4. Butterfly effect for $\tilde{q}=0.05$. All other parameters remain as above.

converges to positive values. It has also been verified that periodic states present negative values accordingly.

\section{Conclusions}

A system for Malkus' waterwheel was proposed and the difficulties of it having a piecewise term were presented. On the basis of calculating a global Lyapunov exponent based on the average of many local ones, we proposed a simplified Jacobian. A relation between the local Lyapunov exponents and the angular velocity of the waterwheel was presented, along with its physical explanation. We then found that local Lyapunov exponents under a chaotic regime do not have positive signs in this system, and exposed the reasons for this to happen. Finally, a numerical method to detect long term divergence, and identify its corresponding Lyapunov exponent was presented, and resulted in coherent results that correctly make the distinction between chaotic and periodic regimes.

As we stated previously, it is the repeated top bucket transitions that create divergence in the long run. Therefore, the more buckets, the more frequency of these transitions we will have. The Lorenz equations were derived from Malkus' waterwheel in assumption of having a very high number 

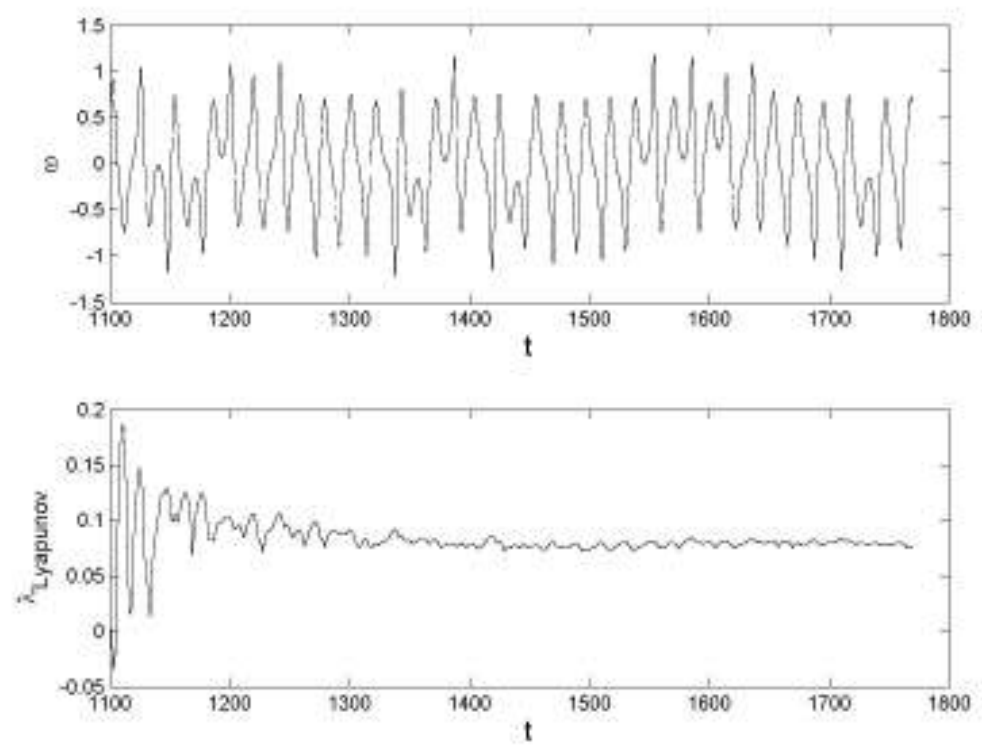

Fig. 5. Global Lyapunov exponent obtained using [6, 2] where $\tilde{q}=0.02$.

of buckets. This is why Lorenz presents immediate divergence in its chaotic regime while the waterwheel takes longer. An extra nonlinearity in the mass equation could close this gap between Lorenz and the discrete waterwheel.

\section{Bibliography}

[1] David Becerra and Valery Tereshko. Chaotic waterwheel: Discrete vs continuous mass representation. Chaos2008 Proceedings, 1, 2008.

[2] Giancarlo Benettin, Luigi Galgani, and Jean-Marie Strelcyn. Kolmogorov entropy and numerical experiments. Phys. Rev. A, 14:2338-2345, 1976.

[3] M. Kolr and G. Gumbs. Theory for the experimental observation of chaos in a rotating waterwheel. Physical Review A, 45(2):626-637, 1992.

[4] E. N. Lorenz. Deterministic non-periodic flows. J. Atmos. Sci, 1963.

[5] W. V. R. Malkus. Non-Periodic convection at high and low prandtl number. Mem. Soc. R. Sci. Liege, 6(IV):125, 1972.

[6] A. Wolf, J. B. Swift, H. L. Swinney, and J. A. Vastano. Determining lyapunov exponents from a time series. Physica D, 16(3):285-317, 1985. 